\title{
Mechanism of the Stereoselective $\alpha$-Alkylation of Aldehydes Driven by the Photochemical Activity of Enamines
}

\author{
Ana Bahamonde ${ }^{\dagger}$ and Paolo Melchiorre ${ }^{*, \dagger, t}$ \\ ${ }^{\dagger}$ ICIQ-Institute of Chemical Research of Catalonia, The Barcelona Institute of Science and Technology, Avinguda Països Catalans \\ 16, 43007 Tarragona, Spain \\ ${ }^{\ddagger}$ ICREA-Catalan Institution for Research and Advanced Studies, Passeig Lluís Companys 23, 08010 Barcelona, Spain
}

Supporting Information

ABSTRACT: Herein we describe our efforts to elucidate the key mechanistic aspects of the previously reported enantioselective photochemical $\alpha$-alkylation of aldehydes with electronpoor organic halides. The chemistry exploits the potential of chiral enamines, key organocatalytic intermediates in thermal asymmetric processes, to directly participate in the photoexcitation of substrates either by forming a photoactive electron donor-acceptor complex or by directly reaching an electronically excited state upon light absorption. These photochemical mechanisms generate radicals from closedshell precursors under mild conditions. At the same time, the ground-state chiral enamines provide effective stereochemical

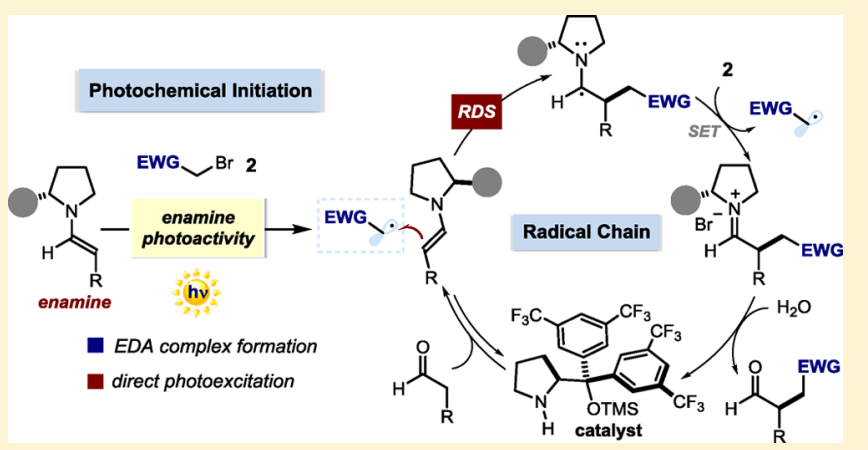
control over the enantioselective radical-trapping process. We use a combination of conventional photophysical investigations, nuclear magnetic resonance spectroscopy, and kinetic studies to gain a better understanding of the factors governing these enantioselective photochemical catalytic processes. Measurements of the quantum yield reveal that a radical chain mechanism is operative, while reaction-profile analysis and rate-order assessment indicate the trapping of the carbon-centered radical by the enamine, to form the carbon-carbon bond, as rate-determining. Our kinetic studies unveil the existence of a delicate interplay between the light-triggered initiation step and the radical chain propagation manifold, both mediated by the chiral enamines.

\section{INTRODUCTION}

Ground-state enamine chemistry has been extensively explored since the 1950s. Following pioneering studies by Gilbert Stork, organic chemists have exploited enamines' nucleophilic character to trap electrophiles and develop useful two-electron polar processes. ${ }^{1}$ Successively, chiral enamines I, generated in situ upon condensation of aldehydes 1 with secondary amine catalysts, have been recognized as key intermediates of organocatalytic enantioselective reactions (Figure 1a)., Single-electron oxidation of ground-state chiral enamines by a chemical oxidant has also been found to render $3 \pi$-electron radical cation intermediates amenable to a range of unique open-shell reaction manifolds (singly occupied molecular orbital (SOMO) activation, Figure $1 \mathrm{~b}) .{ }^{4}$ Overall, the past 15 years have witnessed the extensive use of the ground-state reactivity of enamines for the stereoselective functionalization of carbonyl compounds.

Recently, our research laboratories demonstrated that the synthetic potential of chiral enamines is not limited to the ground-state domain, but can be further expanded by exploiting their photochemical activity. We revealed the previously hidden ability of enamines to actively participate in the photoexcitation of substrates and trigger the formation of reactive open-shell species from organic halides. ${ }^{6}$ At the same time, ground-state chiral enamines can provide effective stereochemical control over the enantioselective radical-trapping process. This strategy, where stereoinduction and photoactivation merge in a sole chiral organocatalyst, enables light-driven enantioselective transformations that cannot be realized using the thermal reactivity of enamines. Specifically, we used this approach to develop the $\alpha$-alkylation of aldehydes ${ }^{7}$ with electron-deficient benzyl and phenacyl bromides (Figure 1c) ${ }^{6 a}$ and bromomalonates 2c (Figure 1d). ${ }^{6}$ The reactions were conducted at ambient temperature using household compact fluorescence light (CFL) bulbs as the light source.

At first glance, both processes depicted in Figure 1c,d seem to be classical substitution reactions of enamines proceeding through an $\mathrm{S}_{\mathrm{N}} 2$ manifold. However, they do not proceed at all without light illumination. Crucial for reactivity was the ability of enamines to trigger the photochemical formation of radicals from the alkyl halides 2 under mild conditions. Despite the superficial similarities between the two chemical transformations, they profoundly diverge in the radical generation mechanism. The first strategy (Figure 1c) relied on the formation of photon-absorbing electron donor-acceptor (EDA) complexes, ${ }^{8}$ generated in the ground state upon association of the electron-rich enamine I with electron-

Received: May 11, 2016

Published: June 6, 2016 
Ground State Reactivity

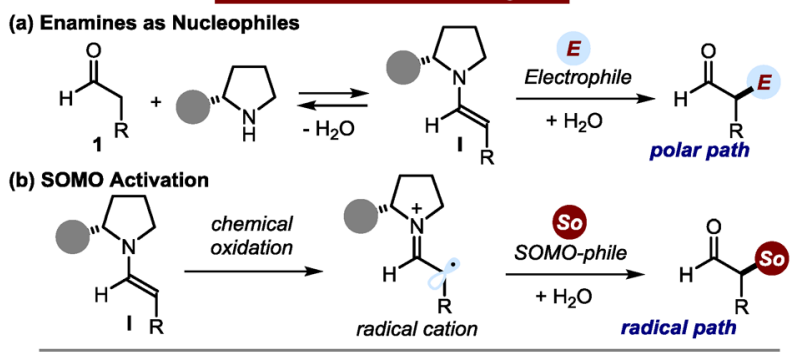

Excited State Domain

(c) Enamines as Donors in Photoactive EDA Complexes

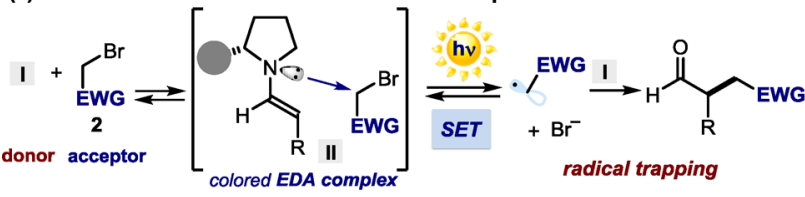

(d) Photochemistry of Enamines in the Excited State

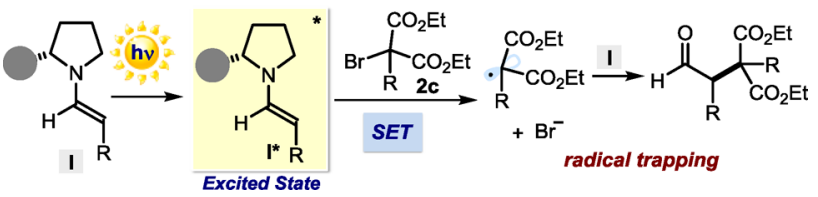

Figure 1. Enamine reactivity domains. Ground-state reactivity: enamines as (a) nucleophiles in traditional polar processes and (b) radical precursors upon single-electron chemical oxidation. Excitedstate domain: enamines can drive the photochemical generation of radicals by (c) inducing the formation of ground-state, photoactive EDA complexes and (d) acting as a photoinitiator upon direct light excitation. SET $=$ single-electron transfer. So $=$ SOMO-phile that can intercept the enamine radical cation. The gray circle represents the chiral organic catalyst scaffold.

deficient benzyl and phenacyl bromides. Visible light irradiation of the colored EDA complex II induced a single-electron transfer (SET), allowing access to the reactive open-shell intermediates. In the second approach (Figure 1d), we used the capability of the chiral enamine I to directly reach an electronically excited state $\left(\mathbf{I}^{*}\right)$ upon light absorption and then to act as an effective photoinitiator. SET reduction of the bromomalonate $2 \mathrm{c}$ induced the formation of the carboncentered radical.

In this paper, we detail how a combination of photophysical investigations, nuclear magnetic resonance (NMR) spectroscopy, kinetic studies, and quantum yield measurements revealed further mechanistic analogies and striking differences for these enamine-mediated photochemical enantioselective alkylations of aldehydes with electron-poor alkyl halides. From a broader perspective, these studies explain how it is possible to translate the effective tools governing the success of ground-state asymmetric enamine catalysis into the realm of photochemical reactivity, ${ }^{9}$ thus providing novel reactivity frameworks for conceiving light-driven enantioselective catalytic processes. ${ }^{10}$

\section{RESULTS AND DISCUSSION}

Our recent studies ${ }^{6}$ established that enamines I can interact with visible light in two different ways, serving either as donors in photoactive EDA complex formation (Figure 1c) or as photoinitiators upon direct excitation (Figure 1d). As the prototypical reactions for mechanistic analysis, we selected the alkylations of butanal (1a) with 2,4-dinitrobenzyl bromide (2a; Figure $2 \mathrm{a})$, phenacyl bromide (2b; Figure $2 \mathrm{~b})$, and diethyl bromomalonate (2c; Figure $2 \mathrm{c}$ ), all promoted by the commercially available diarylprolinol silyl ether catalyst $\mathbf{A}^{11}$ $(20 \mathrm{~mol} \%){ }^{12}$ The reactions with $\mathbf{2 a}$ and $\mathbf{2 b}$ are representative of the EDA complex activation strategy, ${ }^{6 a, b}$ while the chemistry in Figure $2 \mathrm{c}$ is triggered by the direct photoexcitation of the enamine. ${ }^{6 c}$ For all the processes, and in accordance with the original reports, we confirmed that irradiation by a household $23 \mathrm{~W}$ CFL bulb was needed to achieve the alkylation products $3 \mathbf{a}-3 \mathbf{c}$ in high yield and enantioselectivity. ${ }^{13}$ The careful exclusion of light completely suppressed the reactions, confirming their photochemical nature. The inhibition of the reactivity was also observed under an aerobic atmosphere or in the presence of TEMPO ( 1 equiv), the latter experiment indicating a radical mechanism.

Along with these similarities, the light-triggered reactions in Figure 2 showed striking differences too. When the experiments were conducted under illumination by a $300 \mathrm{~W}$ xenon lamp (a)<smiles>CCC(=O)CBr</smiles>

EDA Complex Activation Strategy

(b)

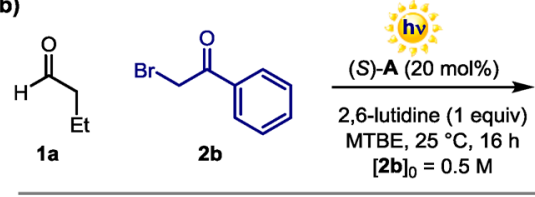

(c) Direct Photoexcitation of Enamines

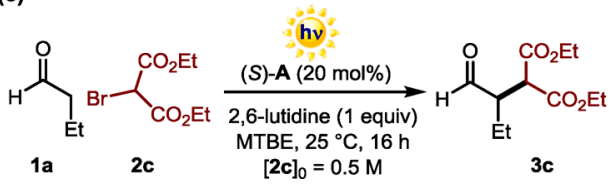

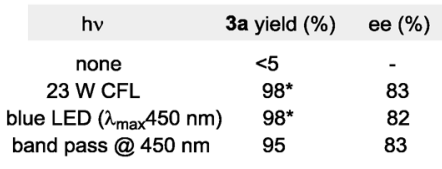

3a

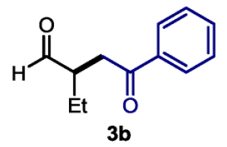

hv

none

$23 \mathrm{~W}$ CFL

3b yield (\%) ee (\%)

$81^{*}$ 

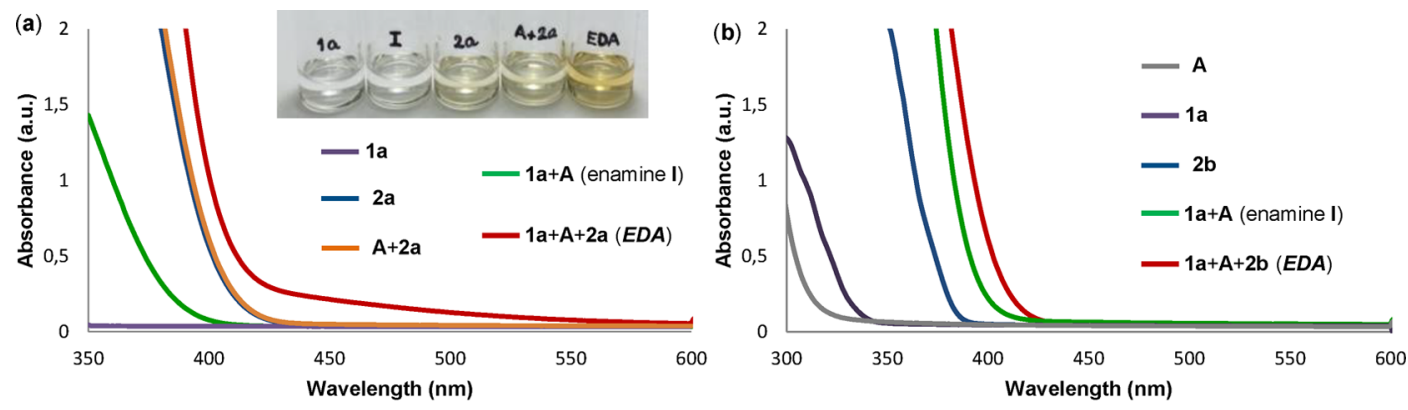

(c)
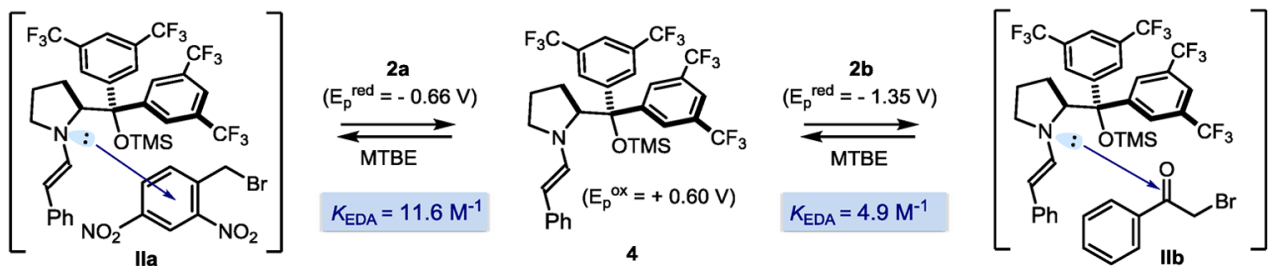

(d)
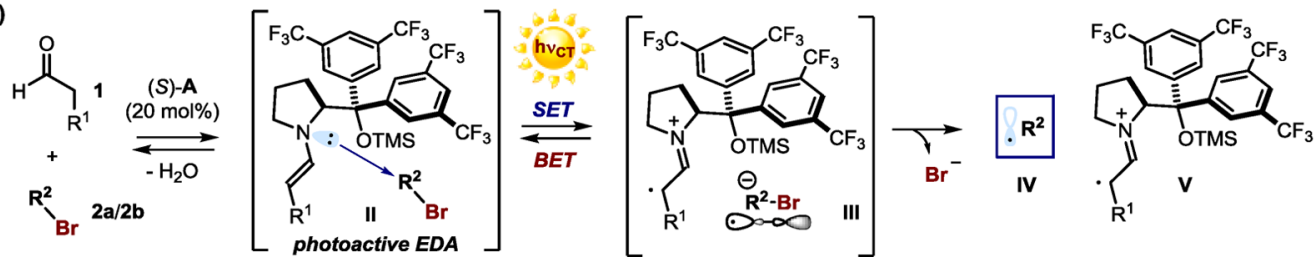

Figure 3. (a) Optical absorption spectra, recorded in MTBE in $1 \mathrm{~mm}$ path quartz cuvettes using a Shimadzu 2401PC UV-vis spectrophotometer, and visual appearance of the separate reaction components and of the colored EDA complex in the alkylation of 2,4-dinitrobenzyl bromide (2a). $[\mathbf{1 a}]=1.5 \mathrm{M},[\mathbf{2 a}]=0.5 \mathrm{M}$, and $[\mathbf{A}]=0.1 \mathrm{M}$. (b) Optical absorption spectra in MTBE for the alkylation with phenacyl bromide $(\mathbf{2 b})$. [1a $]=1.5 \mathrm{M}$ and $[\mathbf{2 b}]=[\mathbf{A}]=0.2 \mathrm{M}$. (c) Investigating the formation of the EDA complexes in MTBE using the preformed enamine 4 . $K_{\mathrm{EDA}}$ is the association constant for the EDA complex formation. $E_{\mathrm{p}}^{\text {red }}$ for $\mathbf{2 a}$ and $\mathbf{2 b}$ (irreversible reduction) and $E_{\mathrm{p}}^{\text {ox }}$ for $\mathbf{4}$ (irreversible oxidation) measured by cyclic voltammetry vs $\mathrm{Ag} / \mathrm{Ag}^{+}$in $\mathrm{CH}_{3} \mathrm{CN}$. (d) Visible-light-triggered generation of the electrophilic carbon-centered radical IV and the $\alpha$-iminyl radical cation $\mathbf{V}$ using the enamine-based EDA complex strategy. $h \nu_{\mathrm{CT}}=$ charge-transfer transition energy. ${ }^{16} \mathrm{BET}=$ back electron transfer.

equipped with a cutoff filter at $385 \mathrm{~nm}$ and a band-pass filter at $400 \mathrm{~nm}$ (irradiation at $\lambda \geq 385 \mathrm{~nm}$ and $\lambda=400 \mathrm{~nm}$, respectively), the reactivity of the three processes remained unaltered. However, the use of a band-pass filter at $450 \mathrm{~nm}$ or a blue light-emitting diode (LED) $\left(\lambda_{\max }\right.$ at $\left.450 \mathrm{~nm}\right)$ completely inhibited the reaction with diethyl bromomalonate $(2 \mathrm{c})$. In sharp contrast, the enamine-mediated alkylations with $\mathbf{2 a}$ and 2b were not affected. We decided to conduct spectroscopic investigations to rationalize the different light-wavelength/ reactivity correlation profiles while elucidating the origins of the enamine's photochemical activity.

Spectroscopic Studies. Immediately after mixing a methyl tert-butyl ether (MTBE) solution of the enamine, generated in situ upon condensation of butanal (1a) (3 equiv) with $20 \mathrm{~mol}$ $\%$ catalyst A, with 2,4-dinitrobenzyl bromide (2a) (1 equiv), we observed that the achromatic solution turned to a marked yellow color (Figure 3a). This observation raised the question of how the color developed.

The appearance of strong color on bringing together two colorless organic compounds is not uncommon. In 1952, this phenomenon inspired Robert Mulliken to formulate the charge-transfer theory. ${ }^{8 \mathrm{c}}$ According to this theory, the association of an electron-rich substrate with a low ionization potential (such as an enamine) ${ }^{14}$ with an electron-accepting molecule with a high electronic affinity ${ }^{15}$ (such as electrondeficient benzyl and phenacyl bromides) can bring about the formation of a new molecular aggregation in the ground state: the electron donor-acceptor complex. EDA complexes are characterized by physical properties that differ from those of the separated substrates. This is because new molecular orbitals form, emerging from the electronic coupling of the donor and acceptor frontier orbitals (highest occupied molecular orbital (HOMO)/lowest unoccupied molecular orbital (LUMO)). EDA formation is accompanied by the appearance of a new absorption band, the charge-transfer band $\left(h \nu_{\mathrm{CT}}\right)$, associated with an intracomplex transfer of a single electron (SET) from the donor to the acceptor. In many cases, the energy of this transition lies within the visible range. ${ }^{16}$ This is what happened when the enamine, generated in situ upon condensation of catalyst $\mathbf{A}$ and $\mathbf{1 a}$, was mixed with both 2,4-dinitrobenzyl bromide (2a) $\left(E_{\mathrm{p}}^{\text {red }}=-0.66 \mathrm{~V}\right.$ vs $\mathrm{Ag} / \mathrm{Ag}^{+}$in $\left.\mathrm{CH}_{3} \mathrm{CN}\right)$ and phenacyl bromide $(\mathbf{2 b}) \quad\left(E_{\mathrm{p}}^{\text {red }}=-1.35 \mathrm{~V}\right.$ vs $\mathrm{Ag} / \mathrm{Ag}^{+}$in $\mathrm{CH}_{3} \mathrm{CN}$ ). Indeed, the optical absorption spectra showed a bathochromic displacement in the visible spectral region, where none of the substrates absorb (red lines, Figures 3a,b). The new absorption bands, which in the case of $2 a$ can reach the green region of the visible range $(550 \mathrm{~nm})$, cannot be accounted for by the addition of the absorption of the separate compounds, which can barely absorb visible light.

To further examine the implication of the enamine in the formation of photoactive EDA complexes, we synthesized the enamine $4\left(E_{\mathrm{p}}{ }^{\text {ox }}=+0.60 \mathrm{~V}\right.$ vs $\mathrm{Ag} / \mathrm{Ag}^{+}$in $\left.\mathrm{CH}_{3} \mathrm{CN}\right)$, prepared by condensation of catalyst $\mathbf{A}$ and 2-phenylacetaldehyde ${ }^{17}$ in the presence of molecular sieves. Upon isolation, 4 was mixed with electron acceptors $\mathbf{2 a}$ and $\mathbf{2 b}$ (Figure $3 \mathbf{c}$ ). Using Job's method ${ }^{18}$ of continuous variations, we readily established a molar donor:acceptor ratio of 1:1 in solution for both colored EDA complexes IIa and IIb, respectively (details in section D of the 
Supporting Information). Concomitantly, an association constant $\left(K_{\mathrm{EDA}}\right)$ of $11.56 \pm 0.02 \mathrm{M}^{-1}$ for the complex IIa and $4.9 \pm 0.1 \mathrm{M}^{-1}$ for IIb in MTBE was determined by spectrophotometric analysis using the Benesi-Hildebrand method. ${ }^{19}$

The light-wavelength/reactivity correlation for the photochemical alkylations of butanal with $\mathbf{2 a}$ and $\mathbf{2 b}$ (parts $\mathbf{a}$ and $\mathbf{b}$, respectively, of Figure 2) can be rationalized on the basis of the photoactivity of the enamine-based EDA complexes IIa and IIb (their absorption spectra, which are similar to the EDA absorption in Figure 3a,b, are reported in Figure S6 in the Supporting Information). Absorption of low-energy photons, including visible light, can induce an electron transfer to occur, leading to the chiral ion pair III (Figure 3d). Critical to reaction development is the presence of the bromide anion within the radical anion partner in III. The bromide, acting as a suitable leaving group, triggers an irreversible fragmentation event ${ }^{20}$ rapid enough to compete with a possible back electron transfer (BET), which would unproductively restore the ground-state EDA complex II instead. ${ }^{21}$ This fragmentation productively renders two reactive radical intermediates (the electrophilic carbon-centered radical IV and the $\alpha$-iminyl radical cation $\mathbf{V}$ ) which can initiate synthetically useful transformations, i.e., the alkylation of aldehydes. The enamine-based EDA complex activation strategy thus provides ready access to open-shell reactive species under very mild conditions and without the need for any external photoredox catalyst.

The enantioselective photochemical alkylation of butanal (1a) with diethyl bromomalonate (2c) showed profoundly different behavior. In addition to the distinct effect the light frequency had on the reactivity (as discussed in Figure 2), we did not observe any color change in the solution, which remained achromatic during the reaction progression. The absence of any photoabsorbing ground-state EDA complex was further confirmed by the optical absorption spectrum of the reaction mixture (red line in Figure 4), which perfectly overlaid

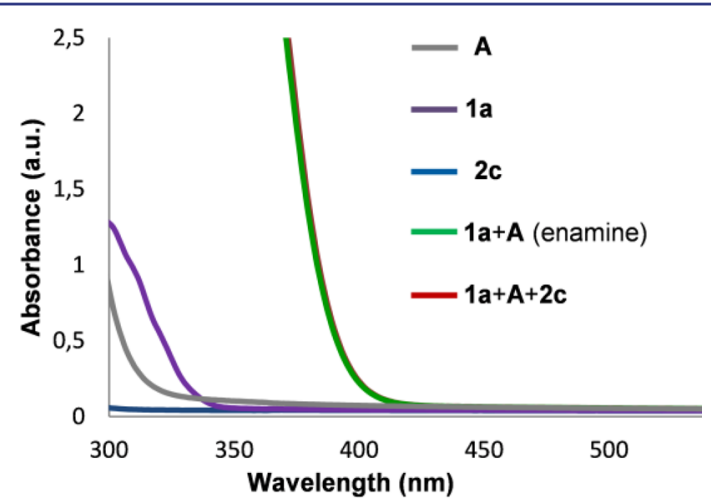

Figure 4. Optical absorption spectra acquired in MTBE in $1 \mathrm{~cm}$ path quartz cuvettes. $[\mathbf{1 a}]=1.5 \mathrm{M},[\mathbf{2 c}]=0.5 \mathrm{M}$, and $[\mathrm{A}]=0.1 \mathrm{M}$.

the absorption of the enamine, generated upon condensation of the catalyst A with 1a (green line in Figure 4). In a separate experiment, we observed that the addition of a large excess of $2 c$ to a solution of enamines did not change the absorption spectra, further excluding any EDA association in the ground state (Figure S13 in the Supporting Information). Closer inspection of the absorption spectrum indicated that the only photoabsorbing compound at $400 \mathrm{~nm}$ (a wavelength suitable for triggering the reaction) was the enamine ${ }^{22}$ (green line in Figure 4, absorption band until $415 \mathrm{~nm}$ ). This observation prompted us to evaluate the possibility that the direct photoexcitation of the enamine could trigger the radical generation from $2 c$. This mechanistic scenario was consonant with the experiment performed using a band-pass filter at 450 $\mathrm{nm}$ (a wavelength that could not be absorbed by the enamine), since a complete inhibition of the reaction was observed (Figure 2c). The implication of the enamine within the photochemical regime was unambiguously established by Stern-Volmer quenching studies. As detailed in our original study, ${ }^{6 c}$ we recorded the emission spectra of enamine 4 upon excitation at $365 \mathrm{~nm}$. The excited state of 4 and its emission were effectively quenched by bromomalonate $2 \mathrm{c}$ (see section E2 in the Supporting Information for details).

These observations indicate that the photochemical activity of chiral enamines and their potential for light-induced radical generation are not limited to the formation of ground-state EDA complexes. As detailed in Figure 5, the enamine I, upon

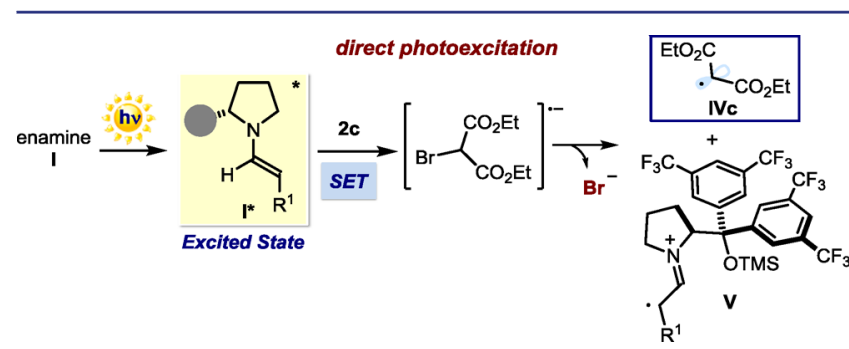

Figure 5. Radical generation strategy based on the direct photoexcitation of the chiral enamine I. The gray circle represents the chiral organic catalyst scaffold.

light absorption, can reach an electronically excited state $\left(\mathbf{I}^{*}\right)$ and act as a photoinitiator, triggering the formation of the electron-deficient radical IVc through the reductive cleavage of the bromomalonate $\mathrm{C}-\mathrm{Br}$ bond via an SET mechanism ${ }^{23}$ $\left(E_{\mathrm{p}}{ }^{\text {red }}(\mathbf{2 c})=-1.69 \mathrm{~V}\right.$ vs $\mathrm{Ag} / \mathrm{Ag}^{+}$in $\left.\mathrm{CH}_{3} \mathrm{CN}\right)$. The reduction potential of the excited enamine was estimated as $<-2.0 \mathrm{~V}$ (vs $\mathrm{Ag} / \mathrm{Ag}^{+}$in $\mathrm{CH}_{3} \mathrm{CN}$ ) on the basis of electrochemical and spectroscopic measurements (see section E3 in the Supporting Information for details). ${ }^{24}$ In analogy with the EDA complex activation (Figure 3d), here too the SET event leads to both an electrophilic radical, IV, and the $\alpha$-iminyl radical cation $\mathbf{V}$.

Quantum Yield Measurements and the Proposed Mechanisms. Photophysical investigations established that in situ generated chiral enamines can use two different photochemical mechanisms to provide open-shell species from organic halides $\mathbf{2 a}-\mathbf{2} \mathbf{c}$ while avoiding the need for any external photoredox catalyst. We then focused on the nonphotochemical steps inherent to the enantioselective alkylation of butanal (1a). As depicted in Figures $3 \mathrm{~d}$ and 5, the enamine-mediated photochemical pathways bring about the formation of two radical species: the chiral radical cation $\mathbf{V}$ and the electrophilic radicals IV. A stereocontrolled radical-radical coupling of IV and $\mathbf{V}$ can be invoked to account for the formation of the new carbon-carbon bond and the $\alpha$-carbonyl stereogenic center within the final products $\mathbf{3 a}-\mathbf{3 c}$ (Figure $6 \mathrm{a}$ ). This mechanistic framework would require an enamine-mediated photochemical event for every molecule of product generated.

It must be noted, however, that many radical reactions generally proceed through self-propagating radical chain pathways. ${ }^{25}$ In chain processes, product formation occurs through propagation steps that convert the open-shell intermediate (originating from the substrate precursor) into 


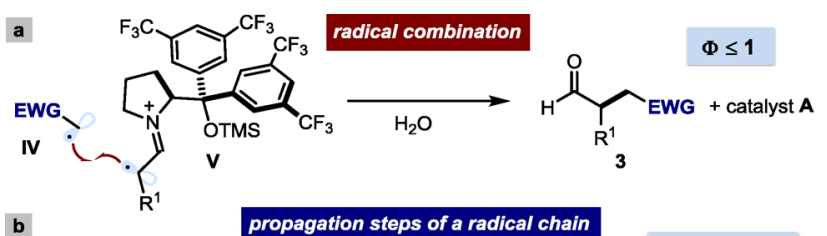

b

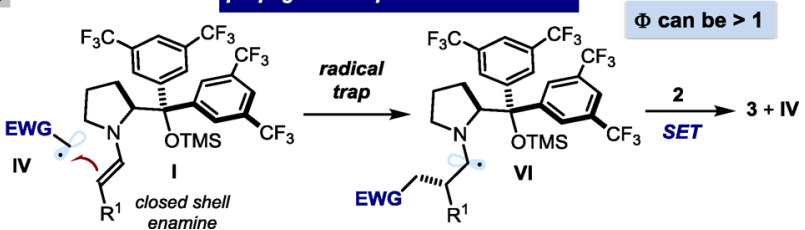

Figure 6. Possible pathways for the nonphotochemical steps of the model reactions: (a) in-cage radical-radical coupling and (b) radical chain propagation manifold. The open-shell intermediates $\mathbf{V}$ and IV are generated through the photochemical activity of the enamines, as detailed in Figures 3d and 5. EWG = electron-withdrawing group. $\Phi=$ overall quantum yield of the alkylation. See ref 28 for an explanation of the quantum yield values.

the final product while regenerating the chain-propagating radical. Reactions will occur if the propagation sequence is rapid enough in comparison with possible termination pathways, and if there is a suitable mode of initiation (that is, effective radical formation from a closed-shell substrate). In our case (Figure 6b), a chain propagation sequence can be envisaged such that the nucleophilic ground-state enamine I would trap the photochemically generated electrophilic radical IV to form the $\alpha$-amino radical VI. Since $\alpha$-aminoalkyl radicals are known to be strong reducing agents, ${ }^{26} \mathrm{VI}$ would induce the reductive cleavage of the electron-poor alkyl bromide 2 through an outer-sphere SET process, thereby regenerating the radical IV while releasing the product $\mathbf{3}$ and the amino catalyst A (more mechanistic details are discussed in Figure 7). In this scenario, the enamine-based photochemical radical generation strategies, which afford radicals IV and $\mathbf{V}$, would serve only to initiate a radical self-propagating chain process.

To help distinguish between the two mechanisms, we determined the quantum yield $(\Phi)^{27}$ of the model reactions, which defines the moles of product formed per moles of photons absorbed by the system. ${ }^{28}$ Using potassium ferrioxalate as the actinometer, we measured quantum yields of 25,20 , and 20 for the reactions in $\mathrm{CH}_{3} \mathrm{CN}^{29}$ with $\mathbf{2 a}, \mathbf{2 b}$, and $\mathbf{2} \mathbf{c}{ }^{30}$ respectively ( $\lambda=450 \mathrm{~nm}$ for $2 \mathbf{a}$ and $2 \mathbf{b}$ and $400 \mathrm{~nm}$ for $2 \mathbf{c}$ ). These results are consonant with a self-propagating radical chain mechanism as the main reaction pathways for the three enamine-mediated photochemical alkylations of butanal under study. The measured quantum yields $\left(\Phi_{\text {measured }}\right)$ refer to the overall reactions. As such, these values do not take into account any possible nonproductive energy-wasting processes, ${ }^{31}$ including parasitic quenching by energy or electron transfer as well as unimolecular decay processes, which do not lead to product formation but which affect the efficiency of photoinitiation. To better estimate the actual chain length of the reactions, we measured the quantum yield of the initiation step, ${ }^{32}$ determining a $\Phi_{\text {initiation }}$ of $0.77,0.68$, and 0.11 for $2 \mathbf{a}, 2 \mathbf{b}$, and $2 c$, respectively ( $\lambda=450 \mathrm{~nm}$ for $\mathbf{2 a}$ and $\mathbf{2 b}$ and $400 \mathrm{~nm}$ for $\mathbf{2 c}$, details in sections G2 and G4 of the Supporting Information). Taking these data into account, the actual chain lengths of the model reactions $\left(\Phi_{\text {estimated }}=\Phi_{\text {measured }} / \Phi_{\text {initiation }}\right)$ are consid-

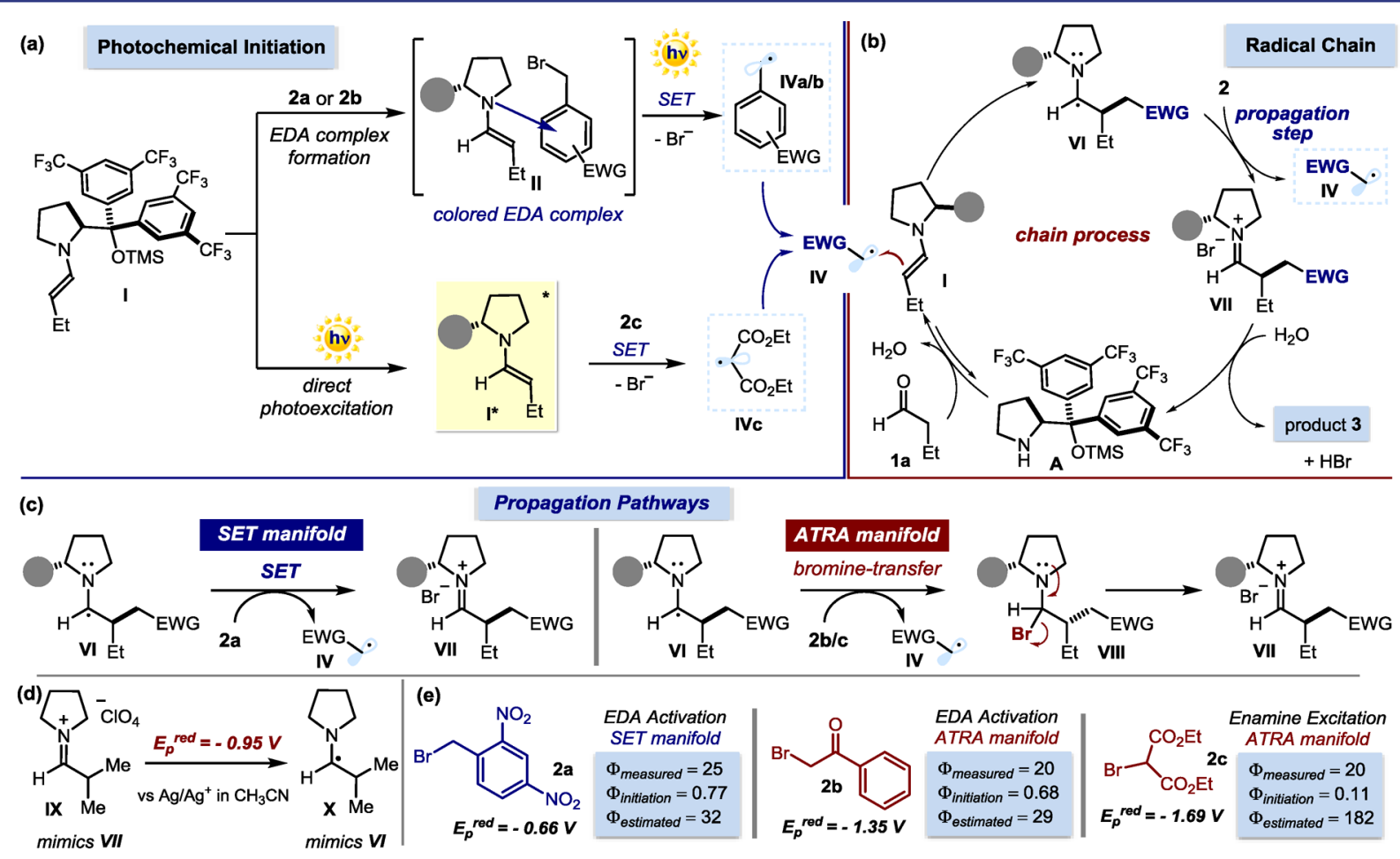

Figure 7. Chain propagation manifold underlying the mechanism of the photochemical enamine-mediated enantioselective $\alpha$-alkylation of butanal. (a) The initiation event, which generates the electrophilic radicals IV, is driven by the photochemical activity of the enamines (EDA complex formation or direct photoexcitation), while (b) the chain process is triggered by the radical trapping by the enamine I. (c) Two possible propagation pathways as driven either by the SET reduction of $\mathbf{2}$ or by the bromine atom transfer from $\mathbf{2}$ involving the key $\alpha$-amino radical VI intermediate. (d) Evaluating the redox potential of the crucial $\alpha$-aminoalkyl radical of type VI. (e) Summary of the quantum yield measurements for the three model photochemical reactions. The gray circle represents the chiral scaffold of the organic catalyst $\mathbf{A}$. 
erably longer, with a lower limit of 32,29 , and 182 for $\mathbf{2 a}, \mathbf{2 b}$, and $2 c$, respectively.

Figure 7 details the general mechanism proposed for the alkylation of butanal with 2,4-dinitrobenzyl bromide (2a), phenacyl bromide (2b), and diethyl bromomalonate (2c). They differ in the nature of the light-triggered initiation step, but are characterized by a similar propagation cycle in which the ground-state enamine I traps the photogenerated electrophilic radical IV. Overall, the mechanism exploits the dichotomous reactivity profile of enamines in the ground and excited states. The photochemical activity of the enamines, either by EDA complex activation or by direct excitation, generates radicals IV from the closed-shell intermediates $\mathbf{2 a - 2} \mathbf{c}$ (Figure $7 \mathbf{a}$ ). ${ }^{33}$ This event, by feeding in radicals from outside the chain, serves as the initiation of self-propagating radical chains. The radical trap from the ground-state chiral enamine I forms the new carboncarbon bond while forging the stereogenic center (Figure $7 \mathrm{~b}$ ). Considering the consolidated ability of catalyst $\mathbf{A}$ to infer high stereoselectivity in enamine-mediated polar reactions, ${ }^{11}$ it is no surprise that the addition of the radical IV to I proceeds in a stereocontrolled fashion. Two pathways are feasible for the propagation step (Figure 7c): the $\alpha$-aminoalkyl radicals VI, resulting from the radical trap, can transfer an electron to the starting alkyl halides 2 . This SET process regenerates the chainpropagating radical IV while giving the bromide-iminium ion pair VII, which eventually hydrolyzes to release the product 3 and the amino catalyst A. The outer-sphere SET process is facilitated by the formation of the stable bromide and iminium ions. Alternatively, an atom-transfer mechanism can be envisaged, where the $\alpha$-aminoalkyl radical VI would abstract a bromine atom from 2, regenerating the radical IV while affording an unstable $\alpha$-bromo amine adduct, VIII, ${ }^{34}$ which would eventually evolve to the iminium ion pair VII. This pathway would provide a rare example of enantioselective catalytic atom-transfer radical addition (ATRA), ${ }^{35}$ a historical methodology useful for functionalizing olefins with organic halides.

To discriminate between the possible propagation manifolds, we prepared and isolated the iminium ion IX (Figure 7d), derived from the condensation of pyrrolidine and isobutyraldehyde, which mimics the actual iminium ion intermediate VII involved in the catalytic cycle. VII could not be synthesized because of the steric hindrance of catalyst $\mathbf{A}$ hampering a facile condensation with the aldehydic product 3. Evaluating the redox properties of IX is pertinent since its electrochemical reduction provides access to an $\alpha$-aminoalkyl radical of type VI, the key intermediate of the chain propagation. We measured by cyclic voltammetry a reduction potential $\left(E_{\mathrm{p}}{ }^{\text {red }}\right.$ of $\left.\mathbf{I X}\right)$ of -0.95 $\mathrm{V}$ vs $\mathrm{Ag} / \mathrm{Ag}^{+}$in $\mathrm{CH}_{3} \mathrm{CN}$ (irreversible reduction to give the $\alpha$ aminoalkyl radical $\mathbf{X}$ ). This value means that the $\alpha$-amino radical of type $\mathrm{VI}$ is incapable of reducing either $\mathbf{2 b}$ or $\mathbf{2 c}$ $\left(E_{\mathrm{p}}{ }^{\text {red }}(\mathbf{2} \mathbf{b})=-1.35 \mathrm{~V}\right.$ vs $\mathrm{Ag} / \mathrm{Ag}^{+}$in $\mathrm{CH}_{3} \mathrm{CN} ; E_{\mathrm{p}}{ }^{\text {red }}(\mathbf{2 c})=-1.69$ $\mathrm{V}$ vs $\mathrm{Ag} / \mathrm{Ag}^{+}$in $\mathrm{CH}_{3} \mathrm{CN}$ ), indicating that a bromine-transfer mechanism is likely operative with phenacyl bromide and bromomalonate substrates. In contrast, an SET reduction is the most likely pathway when using $\mathbf{2 a}$, since its potential $\left(E_{\mathrm{p}}^{\mathrm{red}}(\mathbf{2 a})=-0.66 \mathrm{~V}\right.$ vs $\mathrm{Ag} / \mathrm{Ag}^{+}$in $\left.\mathrm{CH}_{3} \mathrm{CN}\right)$ makes an SET reduction from intermediate VI feasible.

Several aspects of the mechanism proposed in Figure 7 deserve comment. The underlying radical chain pathway is not surprising when considering that the transformations closely resemble atom-transfer radical addition (ATRA) processes ${ }^{35}$ or a Kornblum-Russell $S_{\mathrm{RN}} 1$-type alkylation. ${ }^{36}$ The $S_{\mathrm{RN}} 1$ is a process through which nucleophilic substitution is achieved on aromatic and aliphatic compounds that bear a suitable leaving group and that do not react through polar nucleophilic mechanisms. This class of transformations is characterized by an innate chain mechanism involving electron-transfer steps with radical ions as intermediates. In some examples of $S_{\mathrm{RN}} 1$ type reactions, electron-rich olefins, including enamines, ${ }^{37}$ efficiently trap electrophilic radicals. In addition, electronpoor benzyl ${ }^{37}$ bromides are suitable substrates for the $S_{\mathrm{RN}} 1$ reaction manifold. On the other side, bromomalonates and phenacyl bromides ${ }^{34}$ are suitable substrates for ATRA processes, which classically proceed via radical chain mechanisms. $^{35}$

Another aspect to consider is the central role of the chiral amino catalyst A. Although the process is characterized by an innate radical chain, the organic catalyst plays a direct role in product formation. Indeed, $\mathbf{A}$ is essential for the propagation mechanism since it transforms an inactive substrate (the aldehyde 1), which is unsuitable for participating in the radical chain, into the electron-rich chiral enamine I, a key intermediate of the propagation cycle. In addition, the enamine is directly involved in both the stereodefining event and the photochemical initiation. As for the initiation, the fate of the chiral $\alpha$-iminyl radical cation $\mathbf{V}$, emerging from the photoinduced SET to 2 (Figures $3 \mathrm{~d}$ and 5), deserves further comment. Intermediate $\mathbf{V}$ is an unproductive species, since it lies outside of the chain propagation manifold which converts substrates into products. We have obtained evidence that $\mathbf{V}$ is an unstable intermediate which cannot be reduced back to the progenitor enamine I. Instead, the $\alpha$-iminyl radical cation $\mathbf{V}$ collapses to give a variety of degradation products that, despite our efforts, have remained unidentified so far. ${ }^{38}$ Thus, the enamine $I$ serves as a sacrificial initiator of the chain mechanism $^{39}$ since, for any photoinduced SET event, a propagating radical IV is generated while a molecule of the chiral catalyst $\mathbf{A}$ is destroyed via decomposition of the intermediate V. By using both gas chromatography (GC-FID; FID = flame ionization detection) and NMR analyses, ${ }^{40}$ we established that the amount of catalyst A decreases constantly during the photochemical alkylation in correlation with the number of initiation events (further discussions in the following sections). The irreversible cyclic voltammogram of the preformed enamine 4 (Figure $\mathrm{S} 16$ in the Supporting Information) is also congruent with the proposed enamine degradation pathway.

With a clearer mechanistic picture in mind, we decided to perform kinetic studies to better understand the relative importance of the initiation step and the propagation cycle for the overall rate, while establishing the turnover-limiting step of the model photochemical catalytic alkylations. However, before this, we investigated whether the different photochemical pathways available to enamines for initiating the chain process (EDA complex formation vs direct photoexcitation) might have an influence on the enamine formation and its concentration in solution. This matters because the amount of enamine in solution has a direct effect on the kinetic profiles of the reactions, since the enamine is involved in both initiation and chain propagation (Figure 7a,b).

NMR Spectroscopic Studies. The catalytically active enamine intermediate $\mathbf{I}$ is generated via the reversible condensation of the chiral amino catalyst A with butanal (1a) (Figure 8a). This reversible process is characterized by an equilibrium constant $\left(K_{\text {enamine }}=[\mathbf{I}]\left[\mathrm{H}_{2} \mathrm{O}\right] /[\mathbf{1} \mathbf{a}][\mathbf{A}]\right)$. As with 


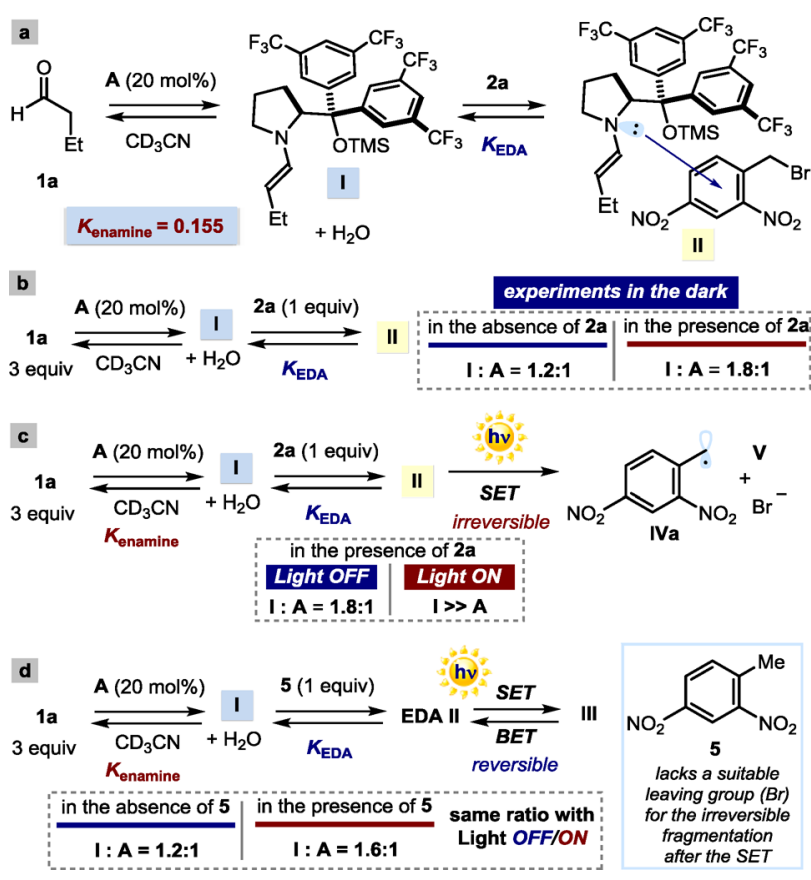

Figure 8. Influence of the EDA complex formation on the amount of enamine in solution. ${ }^{1} \mathrm{H}$ NMR experiments were performed in $\mathrm{CD}_{3} \mathrm{CN}$ at $298 \mathrm{~K}$ using a xenon lamp coupled with a monochromator and equipped with an optical fiber for the in situ illumination of the samples $\left(\lambda=470 \pm 5 \mathrm{~nm}\right.$, irradiance $\left.28.8 \mathrm{~mW} / \mathrm{cm}^{2}\right)$. (a) Equilibrium constant for the enamine $\mathbf{I}$ formation $\left(K_{\text {enamine }}\right.$ measured in $\mathrm{CD}_{3} \mathrm{CN}$ dried over 4 Å molecular sieves) and the following equilibrium to form an EDA complex, II, with 2a $\left(K_{\mathrm{EDA}}\right)$. (b) Effect on the position of equilibrium for enamine formation in the absence and the presence of the EDA acceptor 2a. (c) Effect of light illumination and the irreversible step (triggered by the photoactivity of the EDA complex II) on the concentration of enamine in solution (see Figure $3 \mathrm{~d}$ for more details and the structures of intermediates III and V). (d) Effect of an EDA complex, unable to undergo a photoinduced irreversible SET event, on the enamine concentration. $\mathrm{BET}=$ back electron transfer.

all chemical equilibria, the system follows Le Châtelier's principle. As a consequence, any perturbation of the equilibrium (as induced by a change in concentration, for example) will shift the position of equilibrium to the side that opposes the perturbation. As discussed above (Figure 3c), the formation of the enamine-based EDA complex is also an equilibrium, where $K_{\mathrm{EDA}}$ identifies the association constant. For example, the EDA complex IIa (formed by the association of the preformed enamine $\mathbf{4}$ with 2,4-dinitrobenzyl bromide (2a)) has a $K_{\mathrm{EDA}}$ of $11.6 \mathrm{M}^{-1}$ in MTBE. This scenario suggests that the presence of acceptor 2a can alter the original state of equilibrium for enamine formation. In other words, it can directly influence the relative concentration of free catalyst $\mathbf{A}$ and enamine $\mathbf{I}$ in solution (Figure 8a).

To verify this possibility, we used ${ }^{1} \mathrm{H}$ NMR spectroscopic analysis to investigate the equilibrium of enamine formation under the reaction conditions (Figure 8b). Upon mixing 0.3 mmol of 1a and $0.02 \mathrm{mmol}$ of the amino catalyst $\mathbf{A}$ in $0.5 \mathrm{~mL}$ of anhydrous $\mathrm{CD}_{3} \mathrm{CN}$, both enamine $\mathbf{I}$ and free catalyst $\mathbf{A}$ were detected in a ratio of 1.2:1. An equilibrium constant $\left(K_{\text {enamine }}\right)$ of $0.155 \pm 0.002$ was determined (see section $\mathrm{H} 1$ in the Supporting Information for details). The addition of $0.1 \mathrm{mmol}$ of 2a induced a shift in the position of the equilibrium toward the enamine $\mathbf{I}$, as demonstrated by the 1.8:1 ratio of $\mathbf{I}$ and free catalyst $\mathbf{A}$. This is congruent with the fact that the formation of the EDA complex, by sequestering I, shifts the dynamic equilibrium of enamine formation to the side that reduces the perturbation (in this case, the forward reaction).$^{41}$ Since these studies were made in the absence of light, we then studied the effect of illumination on the dynamic equilibrium system (Figure 8c). We used a xenon lamp coupled with a monochromator, which, by bringing the light in close contact with the NMR tube through an optical fiber, ${ }^{42}$ allowed for the in situ illumination of the samples. When the EDA complex mixture, originally kept in the dark, was irradiated in situ in the NMR spectrometer $(\lambda=470 \pm 5 \mathrm{~nm}$, irradiance $28.8 \mathrm{~mW} /$ $\mathrm{cm}^{2}$ ), a large shift in the position of the enamine equilibrium was immediately observed (3.8:1 ratio of $\mathbf{I}$ to $\mathbf{A}$ after $30 \mathrm{~s}$ of irradiation). After $60 \mathrm{~s}$ of irradiation, the signals of the free catalyst A could no longer be detected, meaning that the system dramatically shifted toward the enamine I. This observation can be reconciled with the photochemical activity of the enaminebased EDA complex II, which, upon excitation, induces the irreversible formation of the electrophilic radical IV (upon fragmentation of the $\mathrm{C}-\mathrm{Br}$ bond within the ion pair III; see Figure 3d) and the unstable $\alpha$-iminyl radical cation $\mathbf{V} .^{40}$ These light-triggered events decrease the concentration of both the enamine and $2 a$, further favoring the forward reactions of the multiple equilibrium systems depicted in Figure 8c.

The importance of the irreversible events that follow the photoinduced SET is corroborated by a similar experiment where $\mathbf{2 a}$ was replaced by 2,4-dinitrotoluene (5) (Figure $8 \mathrm{~d}$ ). 5 can act as an acceptor partner in EDA complex formation with the enamine $\mathrm{I}\left(K_{\mathrm{EDA}}=4.6 \pm 0.1 \mathrm{M}^{-1}\right.$ in MTBE with enamine 4), but it cannot undergo an irreversible fragmentation, since it lacks a suitable leaving group (e.g., the bromine within 2a). In the dark, the addition of 1 equiv of $\mathbf{5}$ to a solution of catalyst $\mathbf{A}$ and butanal (1a) induced a displacement in the equilibrium of the enamine formation, changing the I:A ratio from 1.2:1 to 1.6:1. This is because an EDA complex, II, can be generated, which perturbs the equilibrium of enamine formation. In sharp contrast, illumination did not change the concentration of the enamine I to any extent. This observation is consonant with an unproductive photoinduced SET and a fast back electron transfer (BET) that, by restoring the ground-state EDA complex II, do not influence either the overall equilibrium of the system or the distribution of catalyst $\mathbf{A}$, which is partitioned between the free state and the enamine $\mathbf{I}$.

These experiments were then repeated with the bromomalonate $2 \mathrm{c}$ (results not shown in Figure 8). In this case, the equilibrium of the enamine formation $\left(K_{\text {enamine }}=0.155\right.$ as in Figure $8 \mathrm{a}$ ) was not perturbed by the addition of $2 \mathrm{c}$. This is because the mechanism of initiation is based on the direct photoexcitation of the enamine I and does not involve any preassociation with $2 \mathrm{c}$. Thus, the presence of $2 \mathrm{c}$ does not influence the partitioning of the catalyst $\mathbf{A}$ between the free state and the enamine $\mathbf{I}$.

Kinetic Studies. We then performed kinetic studies to gain a better understanding of the factors governing the photochemical enamine-based alkylations of butanal (1a). In particular, we sought to assess whether the existence of two different initiation methods, but seemingly similar propagation cycles, would bring about distinct or analogous kinetic profiles. The amine-A-catalyzed alkylation of 1a with 2,4-dinitrobenzyl bromide (2a) was chosen as representative of the EDA complex activation strategy (Figure 9a), ${ }^{43}$ while the reaction with diethyl bromomalonate $(2 \mathrm{c})$ exploits the direct photoexcitation of the 
(a) EDA Complex Activation Strategy

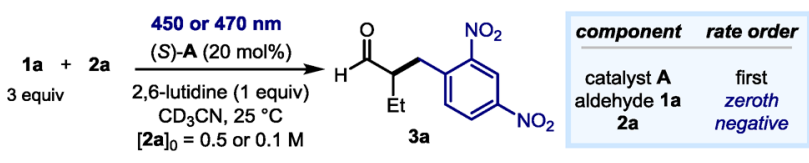

(b) Direct Photoexcitation of Enamines

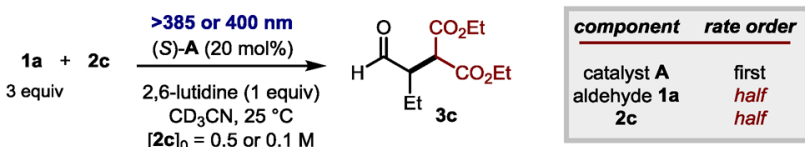

Figure 9. Model reactions used for initial-rate kinetics determined by ${ }^{1} \mathrm{H}$ NMR analysis and the observed rate orders. (a) EDA-complextriggered photochemical alkylation of butanal (1a) with 2,4dinitrobenzyl bromide (2a). (b) Alkylation of 1a with diethyl bromomalonate (2c) driven by the direct photoexcitation of enamines. Reaction conditions: studies performed across a range of concentrations for each reaction component in $\mathrm{CD}_{3} \mathrm{CN}$, irradiation at 450 and $>385 \mathrm{~nm}$ for $\mathbf{2 a}$ and $\mathbf{2 c}$, respectively. The kinetic studies were repeated using in situ ${ }^{1} \mathrm{H}$ NMR spectroscopy $(\lambda=470$ and $400 \mathrm{~nm}$ for 2a and $\mathbf{2 c}$, respectively) to directly monitor the reaction progress. Both approaches gave similar kinetic profiles.

enamine (Figure 9b). Initial rate experiments were performed in acetonitrile as the solvent to avoid the precipitation of the lutidinium bromide, generated during the reaction. ${ }^{29}$ The progress of the two reactions was monitored by ${ }^{1} \mathrm{H}$ NMR analysis using two different approaches (see section $\mathrm{I}$ in the Supporting Information for details). We used a xenon lamp with a band-pass filter at $450 \mathrm{~nm}$ (irradiance $4.7 \mathrm{~mW} / \mathrm{cm}^{2}$ ) to illuminate the EDA-complex-mediated reaction with 2 a (Figure 9a), while a cutoff filter at $385 \mathrm{~nm}$ (irradiation at $\lambda \geq 385 \mathrm{~nm}$, irradiance $300 \mathrm{~mW} / \mathrm{cm}^{2}$ ) was employed for the process with $2 \mathrm{c}$ (Figure 9b). This setup required an independent reaction to be performed for every data point at different times. The initialrate kinetic studies were repeated using in situ ${ }^{1} \mathrm{H}$ NMR spectroscopy to directly monitor the reaction progress. ${ }^{44}$ In this second case, we used a xenon lamp coupled with a monochromator, which allowed for the in situ illumination of the samples. The EDA complex-based reaction with 2 a was irradiated at $470 \mathrm{~nm}$ (irradiance $28.8 \mathrm{~mW} / \mathrm{cm}^{2}$ ), while $400 \mathrm{~nm}$ (irradiance $20.4 \mathrm{~mW} / \mathrm{cm}^{2}$ ) was used for the alkylation chemistry with 2c. Both approaches gave similar and reproducible kinetic profiles.

Figure 9 details the results of our initial-rate kinetic investigations, performed across a range of concentrations for each reaction component. A first-order dependence on the catalyst A was inferred for both the EDA-complex-based process with $\mathbf{2 a}$ (Figure 9a) and the reaction with bromomalonate 2c (Figure 9b). However, striking discrepancies in rate orders were observed in the dependence on butanal (1a) and organic halides $\mathbf{2 a}$ and $\mathbf{2 c}$. The EDA-complexmediated alkylation showed a zeroth-order dependence on the 1a concentration and an unexpected negative fractional order in [2a]. In sharp contrast, the photochemical alkylation of $2 \mathrm{c}$ is characterized by a half-order dependence on both $[\mathbf{1 a}]$ and [2c]. We also explored the effect of water on the kinetic profile of the two processes using both the independent measurement method and in situ NMR approach (details in Figures S31 and S39). No alteration of the kinetic profiles was observed after the addition of either 1 or 2 equiv of $\mathrm{H}_{2} \mathrm{O}$. These results indicate that the iminium ion hydrolysis, which leads to the alkylation product 3 while liberating the catalyst $\mathbf{A}$, is not turnoverlimiting.

We then tried to reconcile the strikingly different kinetic behaviors of the two systems with our previous observations. The zeroth-order dependence on butanal (1a) for the EDAcomplex-mediated alkylation with $2 \mathrm{a}$ implies that the enamine I, generated in situ upon condensation of $\mathbf{A}$ and 1a, is the resting state of catalyst $\mathbf{A}$. This conclusion is consonant with the NMR spectroscopic studies reported in Figure 8b,c indicating that, under the reaction conditions - that is, when the EDA complex between the enamine $\mathbf{I}$ and $\mathbf{2} \mathbf{a}$ is formed and under illumination-the equilibrium position of the enamine formation is completely shifted toward the enamine I. This means that a negligible amount of catalyst $\mathbf{A}$ is available in its free state and, consequently, the concentration of 1a does not affect the formation of the reactive enamine catalytic intermediate. In sharp contrast, our NMR studies established that the equilibrium of the enamine formation is not perturbed by the addition of bromomalonate $2 \mathrm{c}$. In the direct photoexcitation of the enamine $\mathbf{I}$, the amine catalyst $\mathbf{A}$ is partitioned between the free state and the enamine intermediate $I$. Thus, a definitive resting state cannot be identified, with the catalyst concentration shared between different intermediates. This situation is congruent with the observed positive fractional order in [1a] (Figure 9b).

Concerning the reaction rate's dependence on the alkyl halide 2, the negative fractional order in $[\mathbf{2 a}]$ for the EDAcomplex-driven process (Figure 10a) deserves in-depth discussion. As previously mentioned, for any SET event taking place within the photoactive EDA complex (Figure $3 \mathrm{~d}$ and initiation step in Figure 7), a propagating radical, IV, is generated while a molecule of the chiral catalyst $\mathbf{A}$ is destroyed via decomposition of the unstable $\alpha$-iminyl radical cation $\mathbf{V}{ }^{40}$ To verify whether the disappearance of the catalyst was related to the number of initiation events, we followed the evolution of [A] over time across a range of concentrations of $2 a$, which is the acceptor partner in EDA complex formation. Since there is zeroth-order dependence on $[\mathbf{1 a}]$ and due to the fact that we could not detect any trace of catalyst $\mathbf{A}$ in its free state by NMR analysis, we monitored the evolution of $\mathbf{A}$ in our experiments by determining the enamine concentration in solution. ${ }^{41}$ The initial-rate measurements in Figure $10 \mathrm{~b}$ suggest that the decrease in $[\mathbf{A}]$ correlates with the $2 \mathbf{a}$ concentration. If the rate of disappearance of $\mathbf{A}$ is proportional to $[\mathbf{2} \mathbf{a}]^{n}$, then the data should fit eq 1 . As a result, the kinetic data can be plotted as indicated in eq 2 .

$$
\text { disappearance of } \mathbf{A}=-\mathrm{d}[\mathbf{A}] / \mathrm{d} t \propto[\mathbf{2} \mathbf{a}]^{n}
$$

$$
[\mathbf{A}] \propto[\mathbf{2 a}]^{n} t
$$

Equation 2 indicates that, for reactions with the same initial concentrations of amino catalyst $\mathbf{A}$, plots of $[\mathbf{A}]$ versus $[\mathbf{2 a}]^{n} t$ should be superimposable. ${ }^{45}$ Figure $10 \mathrm{c}$ shows such a superimposition for three reactions that have comparable initial concentrations of $\mathbf{A}$ but different concentrations of $2 \mathrm{a}$. In Figure $10 \mathrm{c}$, the overlay found for plots of $[\mathbf{A}]$ versus $[\mathbf{2 a}] t(n=$ 1 in eq 2) establishes a first-order dependence on $[\mathbf{2 a}]$ for the catalyst's disappearance.

The unitary dependence was also observed using in situ NMR monitoring of the reaction progress (see sections F3 and I1 in the Supporting Information for details). Using this approach, we performed two sets of experiments under the same conditions, but using a different intensity of irradiation $(\lambda$ 


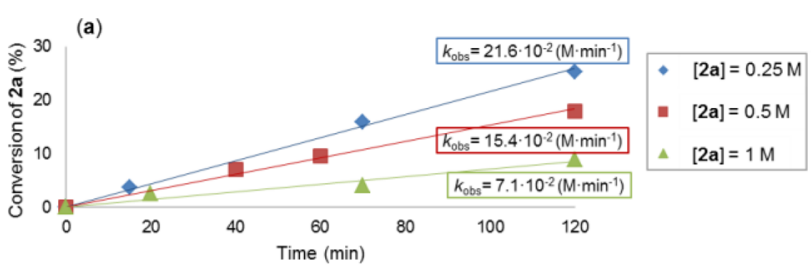

(b) Catalyst A decomposition
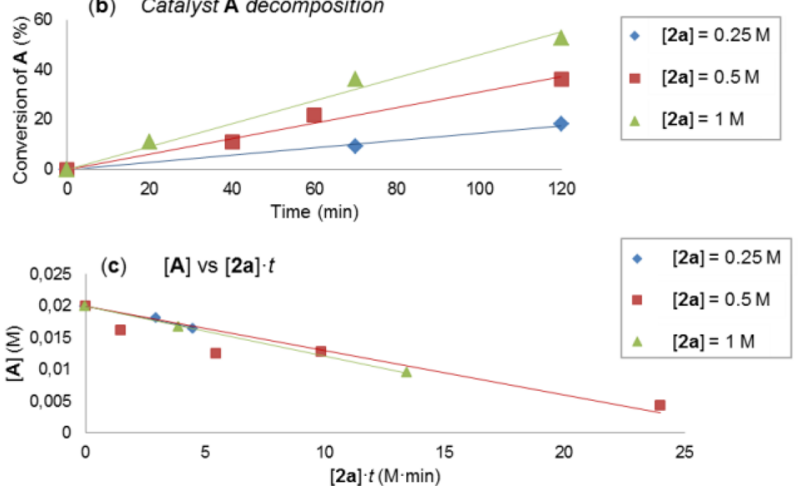

Figure 10. (a) Reaction profiles for different [2a] values showing a negative-order dependence and observed rate constants. $K_{\text {obsd }}$ calculated from the slope of the plots. (b) Evolution of the catalyst concentration for the experiments in (a). We monitored the evolution of $\mathbf{A}$ by determining the enamine concentration in solution. (c) Overlay of plots for the kinetic data in (b) according to eq 2. Progress of the reactions followed by ${ }^{1} \mathrm{H}$ NMR analysis. Each point corresponds to an individual run. Reactions performed in $\mathrm{CD}_{3} \mathrm{CN}$ under illumination by a xenon lamp with a band-pass filter at $450 \mathrm{~nm}$ (irradiance $4.7 \mathrm{~mW} / \mathrm{cm}^{2}$ ). $[\mathbf{1 a}]_{0}=1.5 \mathrm{M}$ and $[\mathbf{A}]_{0}=0.1 \mathrm{M}$. Initial concentrations of 2a: $0.25 \mathrm{M}$ (blue plot); $0.5 \mathrm{M}$ (red plot); $1 \mathrm{M}$ (green plot). The same kinetic profiles have been observed using in situ NMR monitoring of the reaction progress. See section I1 in the Supporting Information.

$=470 \mathrm{~nm}$ for both sets of experiments, but an irradiance of 28.8 $\mathrm{mW} / \mathrm{cm}^{2}$ vs $3.0 \mathrm{~mW} / \mathrm{cm}^{2}$ ). In the latter set of experiments, a lower absolute rate of catalyst decomposition was determined, in consonance with a less effective initiation regime. This observation establishes a direct correlation between the disappearance of catalyst $\mathbf{A}$ and the number of photochemical initiation events, since both the concentration of $2 \mathbf{a}$ and the intensity of light influence the rate of degradation for catalyst A. $^{32}$

We then wanted to measure the real effect of $[2 \mathbf{a}]$ on the rate of alkylation leading to product $3 \mathbf{a}$, discounting the effects of catalyst $\mathbf{A}$ degradation in the initiation regime. Considering the zeroth-order dependence on 1a, the rate equation should read as eq 3 , with $n$ being the rate order with respect to 2 a, overlooking its effect on $[\mathbf{A}]$. In eq 4 , the product formation is normalized by $[\mathbf{A}]$, thus discounting the effect of the catalyst degradation.

$$
\begin{aligned}
& \text { reaction rate }=\mathrm{d}[\mathbf{3} \mathbf{a}] / \mathrm{d} t \propto[\mathbf{A}][\mathbf{2} \mathbf{a}]^{n} \\
& {[\mathbf{3} \mathbf{a}] /[\mathbf{A}] \propto[\mathbf{2} \mathbf{a}]^{n} t} \\
& \log ([\mathbf{3} \mathbf{a}] /[\mathbf{A}] t)=n \log ([\mathbf{2} \mathbf{a}])+\log (c)
\end{aligned}
$$

The rate-order dependence on $[2 \mathbf{a}]$ was calculated by plotting the data according to eq 5 , derived from eq 4 . The order $(n)$ is obtained from the slope of the logarithmic plot displayed in Figure 11a, which indicates a positive fractionalorder dependence on $[2 \mathrm{a}](n \approx 0.4)$, while $c$ is a constant $(c=$
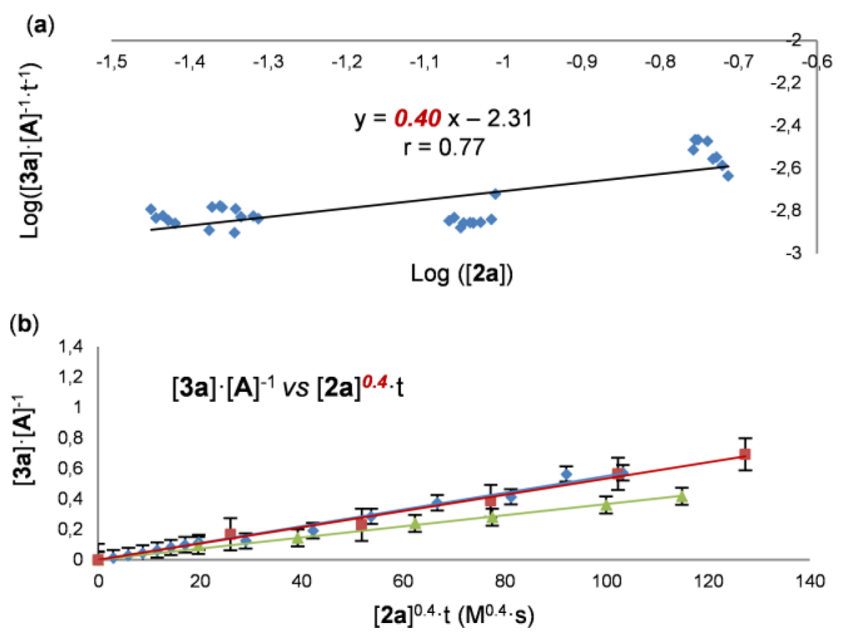

Figure 11. (a) Logarithmic plot according to eq 5 giving a positive fractional-order dependence on $[\mathbf{2 a}](n \approx 0.4)$. (b) Kinetic data according to eq 4 for $n=0.4$. For this fitting, we have used the data obtained by in situ NMR monitoring of the reaction progress, which gives the same kinetic profiles observed in Figure 10 (section I1 in the Supporting Information). This approach has the advantage of providing a larger number of data, thus allowing for a more reliable fitting. Experiments performed in NMR tubes at $298 \mathrm{~K}$ in $\mathrm{CD}_{3} \mathrm{CN}$ using a monochromatic light $\left(\lambda=470 \mathrm{~nm}\right.$, irradiance $\left.28.8 \mathrm{~mW} / \mathrm{cm}^{2}\right)$. $[\mathbf{1 a}]_{0}=0.3 \mathrm{M}$ and $[\mathbf{A}]_{0}=0.02 \mathrm{M}$. Initial concentrations of $2 \mathrm{a}: 0.05 \mathrm{M}$ (blue plot); $0.1 \mathrm{M}$ (red plot); $0.2 \mathrm{M}$ (green plot).

$-2.31)$ given by the $x$-intercept. Figure $11 \mathrm{~b}$ displays the fitting of the kinetic data to eq 4 for $n=0.4$, showing a good overlay.

Overall, eq 6 gives the empirical rate law for the EDAcomplex-mediated alkylation of butanal in Figure 9a, discounting catalyst degradation related to the photochemical initiation.

$$
\text { rate }=\mathrm{d}[\mathbf{3} \mathbf{a}] / \mathrm{d} t=k[\mathbf{A}][\mathbf{2} \mathbf{a}]^{\sim 0.4}
$$

The rate law indicates a turnover-limiting step within the radical chain propagation cycle (see Figure 7 for the general mechanism). If the initiation step was rate-determining, a firstorder dependence with respect to both EDA partners I and 2a would be expected instead. The first-order dependence on catalyst A (whose concentration is equal to the enamine I concentration) suggests that the rate-determining step is the trapping of the electrophilic carbon-centered radical IV from the ground-state chiral enamine I to form the new carboncarbon bond. We would expect higher order dependence on [2a] if the turnover-limiting step were the SET process, which regenerates the chain-propagating radical IV from the $\alpha$ aminoalkyl radicals VI.

The alkylation of $\mathbf{1 a}$ with bromomalonate, driven by the direct excitation of enamine, shows half-order dependence on [2c]. The overall rate equation is then given by eq 7 .

$$
\text { rate }=\mathrm{d}[\mathbf{3} \mathbf{c}] / \mathrm{d} t=k[\mathbf{A}][\mathbf{1} \mathbf{a}]^{0.5}[\mathbf{2} \mathbf{c}]^{0.5}
$$

In analogy with the preceding discussion, the rate-order assessment indicates that the rate-determining step is the enamine trapping the electrophilic radical IV, derived from $2 \mathrm{c}$, to form the carbon-carbon bond (see Figure 7 for the general mechanism).

Notable, no significant degradation of catalyst $\mathbf{A}$ was observed during the alkylation with $2 \mathrm{c}$ within the time frame of interest for the initial-rate measurements using the method of independent experiments. ${ }^{46}$ When the time of irradiation of 
the photochemical alkylation with $2 \mathrm{c}$ was extended, the disappearance of catalyst A became significant. However, using the same $23 \mathrm{~W}$ CFL light source and considering the same time interval, the catalyst degradation was much higher in the alkylations of $\mathbf{2 a}$ and $\mathbf{2 b}$ than the alkylation of $\mathbf{2 c}$ (details in section $\mathrm{F}$ of the Supporting Information). This observation, along with the measured quantum yields of photoinitiation $\left(\Phi_{\text {initiation }}=0.77,0.68\right.$, and 0.11 for $\mathbf{2 a}, \mathbf{2 b}$, and $2 \mathbf{c}$, respectively), suggests that the direct excitation of the enamine $\mathbf{I}$ is a less effective radical generation strategy than the enamine-based EDA complex approach. This scenario can be rationalized on the basis of the bimolecular nature of the initiation mechanism with $2 c$, which requires the excited enamine to encounter $2 c$ for an effective SET. These conditions make an unproductive relaxation of the excited intermediate, which restores the ground-state enamine, more likely. In contrast, the photochemistry underlying the processes with $\mathbf{2 a}$ and $\mathbf{2} \mathbf{b}$ is dominated by EDA complexes. These form in the ground state, holding together the two partners involved in the following photoinduced SET. In this case, the initiation mechanism is based on a more efficient unimolecular process.

\section{CONCLUSIONS}

In summary, we have used a combination of conventional photophysical investigations, NMR spectroscopy, and kinetic studies to elucidate the key mechanistic aspects of the enantioselective photochemical $\alpha$-alkylation of aldehydes with electron-poor organic halides. Quantum yield measurements established that a radical chain propagation mechanism is operative, while reaction profile analysis and rate-order assessment indicated that the trapping of the carbon-centered radical by the enamine is the rate-determining event. Central to these processes is the unique and diverse reactivity of chiral enamines. Their photochemical activity, either by EDA complex activation or by direct excitation, generates radicals from the organic halides $\mathbf{2 a - 2 c}$. This event, by feeding in radicals from outside the chain, serves as the initiation of selfpropagating cycles. The enamine lies at the heart of the propagation cycle too, since it traps the radical to generate an intermediate (the $\alpha$-amino radical VI) which is key for sustaining the chain sequence. We also uncovered how enamine formation and its concentration in solution are directly influenced by the different photochemical pathways available to enamines for initiating the chain process (EDA complex formation vs direct photoexcitation). Overall, the kinetic and spectroscopic investigations allowed us to understand the delicate interplay between the light-triggered initiation step and the radical propagation manifold, suggesting that this approach can be generally applied to the mechanistic elucidation of chain processes. From a broader perspective, this study demonstrates that the synthetic potential of chiral enamines is not limited to the ground-state domain, but can be further expanded by exploiting their photochemical activity, providing novel reactivity frameworks for conceiving light-driven enantioselective catalytic processes.

\section{ASSOCIATED CONTENT}

\section{S Supporting Information}

The Supporting Information is available free of charge on the ACS Publications website at DOI: 10.1021/jacs.6b04871.
Complete experimental procedures, characterization data, and details on kinetic and spectroscopic studies (PDF)

\section{AUTHOR INFORMATION}

\section{Corresponding Author}

*pmelchiorre@iciq.es

\section{Notes}

The authors declare no competing financial interest.

\section{ACKNOWLEDGMENTS}

Financial support was provided by the ICIQ Foundation, MINECO (Project CTQ2013-45938-P and Severo Ochoa Excellence Accreditation 2014-2018, SEV-2013-0319), the AGAUR (Grant 2014 SGR 1059), and the European Research Council (Grant ERC 278541-ORGA-NAUT). A.B. is grateful to the MECD for an FPU fellowship (ref FPU13/02402). We are indebted to the team of the Research Support Area at ICIQ. We thank Dr. Elena Arceo and Dr. Mattia Silvi for preliminary investigations and useful discussions.

\section{REFERENCES}

(1) (a) Stork, G.; Terrell, R.; Szmuszkovicz, J. J. Am. Chem. Soc. 1954, 76, 2029-2030. (b) Stork, G.; Brizzolara, A.; Landesman, H.; Szmuszkovicz, J.; Terrell, R. J. Am. Chem. Soc. 1963, 85, 207-222.

(2) For seminal papers, see: (a) Eder, U.; Sauer, G.; Wiechert, R. Angew. Chem., Int. Ed. Engl. 1971, 10, 496-497. (b) Hajos, Z. G.; Parrish, D. R. J. Org. Chem. 1974, 39, 1615-1621. (c) List, B.; Lerner, R. A.; Barbas, C. F., III J. Am. Chem. Soc. 2000, 122, 2395-2396. (d) Ahrendt, K. A.; Borths, C. J.; MacMillan, D. W. C. J. Am. Chem. Soc. 2000, 122, 4243-4244.

(3) For insightful perspectives on the scientific roots of enaminemediated stereoselective catalytic reactions, see: (a) Barbas, C. F., III Angew. Chem., Int. Ed. 2008, 47, 42-47. (b) List, B. Angew. Chem., Int. Ed. 2010, 49, 1730-1734.

(4) For the seminal reports of SOMO activation, see: (a) Beeson, T. D.; Mastracchio, A.; Hong, J.-B.; Ashton, K.; MacMillan, D. W. C. Science 2007, 316, 582-585. (b) Jang, H.-Y.; Hong, J.-B.; MacMillan, D. W. C. J. Am. Chem. Soc. 2007, 129, 7004-7005. For mechanistic studies, see: (c) Devery, J. J., III; Conrad, J. C.; MacMillan, D. W. C.; Flowers, R. A., II Angew. Chem., Int. Ed. 2010, 49, 6106-6110.

(5) Mukherjee, S.; Yang, J. W.; Hoffmann, S.; List, B. Chem. Rev. 2007, 107, 5471-5569.

(6) (a) Arceo, E.; Jurberg, I. D.; Álvarez-Fernández, A.; Melchiorre, P. Nat. Chem. 2013, 5, 750-756. (b) Arceo, E.; Bahamonde, A.; Bergonzini, G.; Melchiorre, P. Chem. Sci. 2014, 5, 2438-2442. (c) Silvi, M.; Arceo, E.; Jurberg, I. D.; Cassani, C.; Melchiorre, P. J. Am. Chem. Soc. 2015, 137, 6120-6123.

(7) Prior to our studies, similar enamine-mediated enantioselective alkylations of aldehydes were realized using an external photoredox catalyst, which served to generate the radical species from alkyl halides; see: (a) Nicewicz, D. A.; MacMillan, D. W. C. Science 2008, 322, 7780. (b) Shih, H.-W.; Vander Wal, M. N.; Grange, R. L.; MacMillan, D. W. C. J. Am. Chem. Soc. 2010, 132, 13600-13603.

(8) For reviews, see: (a) Foster, R. J. Phys. Chem. 1980, 84, 21352141. (b) Rosokha, S. V.; Kochi, J. K. Acc. Chem. Res. 2008, 41, 641653. For the seminal studies that inspired the EDA complex theory, see: (c) Mulliken, R. S. J. Phys. Chem. 1952, 56, 801-822. (d) Benesi, H. A.; Hildebrand, J. J. J. Am. Chem. Soc. 1949, 71, 2703-2707. (e) Mulliken, R. S. J. Am. Chem. Soc. 1950, 72, 600-608. (f) Keefer, R. M.; Andrews, L. J. J. Am. Chem. Soc. 1950, 72, 4677-4681.

(9) For the use of chiral enolates, generated under phase-transfer conditions, to form chiral photoactive EDA complexes while promoting an enantioselective process, see: Woźniak, Ł.; Murphy, J. J.; Melchiorre, P. J. Am. Chem. Soc. 2015, 137, 5678-5681. 
(10) Despite their synthetic potential, it is challenging to develop enantioselective catalytic variants of visible-light-driven photochemical reactions. For recent reviews discussing the available strategies, see: (a) Brimioulle, R.; Lenhart, D.; Maturi, M. M.; Bach, T. Angew. Chem., Int. Ed. 2015, 54, 3872-3890. (b) Meggers, E. Chem. Commun. 2015, 51, 3290-3301. For selected examples, see: (c) Bauer, A.; Westkamper, F.; Grimme, S.; Bach, T. Nature 2005, 436, 11391140. (d) Brimioulle, R.; Bach, T. Science 2013, 342, 840-843. (e) Alonso, R.; Bach, T. Angew. Chem., Int. Ed. 2014, 53, 4368-4371. (f) Du, J.; Skubi, K. L.; Schultz, D. M.; Yoon, T. P. Science 2014, 344, 392-396. (g) Huo, H.; Shen, X.; Wang, C.; Zhang, L.; Röse, P.; Chen, L.-A.; Harms, K.; Marsch, M.; Hilt, G.; Meggers, E. Nature 2014, 515, 100-103. (h) Kainz, Q. M.; Matier, C. D.; Bartoszewicz, A.; Zultanski, S. L.; Peters, J. C.; Fu, G. C. Science 2016, 351, 681-684.

(11) For a recent review, see: Donslund, B. S.; Johansen, T. K.; Poulsen, P. H.; Halskov, K. S.; Jørgensen, K. A. Angew. Chem., Int. Ed. 2015, 54, 13860-13874.

(12) The reactions required the presence of 1 equiv of a base (2,6lutidine and $\mathrm{NaOAc}$ giving similar results) to perform well. Removing the base additive, trace amounts of the alkylation products 3 were formed $(<15 \%)$, while reagent decomposition along with acidification of the reaction medium was observed. The likely role of 2,6-lutidine is to quench the acid generated during the process, as testified to by the formation of the insoluble lutidinium bromide salt generated during the reaction. The presence of 2,6-lutidine does not contribute to the absorption in the visible region to any extent.

(13) Performing the alkylation with $2 \mathrm{c}$ under the conditions set out in Figure $2 \mathrm{c}$ but in the presence of $0.5 \mathrm{~mol} \% \mathrm{Ru}(\mathrm{bpy}) 3_{3}{ }^{2+}$ as an external photosensitizer significantly increased the reactivity (2-fold reaction rate), in consonance with the occurrence of an additional photoinduced electron-transfer pathway; see ref 7a. The increase in reactivity becomes larger in polar solvents $\left(\mathrm{DMF}, \mathrm{CH}_{3} \mathrm{CN}\right)$, where $\mathrm{Ru}(\mathrm{bpy})_{3}{ }^{2+}$ is completely soluble. Details are reported in the Supporting Information of ref $6 \mathrm{c}$.

(14) Enamines have a low ionization potential. For example, 1-but-1enylpyrrolidine has an IP of $7.2 \mathrm{eV}$; see: Müller, K.; Previdoli, F.; Desilvestro, H. Helv. Chim. Acta 1981, 64, 2497-2507.

(15) The electron-donor property of a donor molecule is indicated by the magnitude of its ionization potential (IP in the gas phase) or its oxidation potential ( $E_{\mathrm{p}}{ }^{\mathrm{ox}}$ in solution). Conversely, the viability of a compound as an acceptor is indicated by the magnitude of its electron affinity (EA in the gas phase) or its reduction potential $\left(E_{\mathrm{p}}{ }^{\text {red }}\right.$ in solution). $E_{\mathrm{p}}{ }^{\text {ox }}$ and $E_{\mathrm{p}}$ red , which can be estimated by cyclic voltammetry measurements, can help in predicting the suitability of a given substrate to undergo EDA complex formation.

(16) According to the Mulliken theory (ref 8c), the charge-transfer transition energy $\left(h \nu_{\mathrm{CT}}\right)$ is given by the following equation: $h \nu_{\mathrm{CT}}=\mathrm{IP}$ - EA - $\omega$, where $\omega$ is the electrostatic interaction of the chargetransfer ion pair (e.g., III in Figure 3d). Qualitatively, the equation predicts that the charge-transfer band of the EDA complexes with a given electron donor (in this case, the enamine) undergoes a bathochromic shift (absorption at longer wavelengths) with increasing EA of the acceptor component. The same effect can be obtained by increasing the electron donicity (decreasing the IP) of the donor.

(17) 2-Phenylacetaldehyde is a competent substrate of the photoorganocatalytic reaction with $\mathbf{2 a}, \mathbf{2} \mathbf{b}$, and $\mathbf{2 c}$, providing full conversion into the corresponding products under the conditions set out in Figure 2. However, a low level of enantioselectivity was observed (below $30 \%$ ee), a consequence of the lability of the resulting benzylic stereocenter.

(18) Job, P. Ann. Chem. 1928, 9, 113-203.

(19) Benesi, H.; Hildebrand, J. J. Am. Chem. Soc. 1949, 71, 27032707.

(20) Costentin, C.; Robert, M.; Savéant, J.-M. Chem. Phys. 2006, 324, $40-56$.

(21) Although the photophysics of EDA complexes have been extensively studied (refs $8 \mathrm{a}$ and $8 \mathrm{~b}$ ), their use in chemical synthesis has found limited applications. This is mainly because the unproductive, back electron transfer (BET), which restores the ground-state EDA complex, is generally faster than other possible processes leading to products. For a pertinent discussion, see: Rathore, R.; Kochi, J. K. Adv. Phys. Org. Chem. 2000, 35, 193-318.

(22) For an additional example of an enamine, formed upon condensation of an aliphatic aldehyde and a secondary amine, which weakly absorbs in the visible region, see: González-Béjar, M.; Peters, K.; Hallett-Tapley, G. L.; Grenier, M.; Scaiano, J. C. Chem. Commun. 2013, 49, 1732-1734.

(23) For examples of photoinduced reductive cleavage of $\mathrm{C}-\mathrm{X}$ bonds in electron-poor alkyl halides via an SET from excited substrates, see: (a) Rico, I.; Cantacuzene, D.; Wakselman, C. Tetrahedron Lett. 1981, 22, 3405-3408. (b) Iwasaki, T.; Sawada, T.; Okuyama, M.; Kamada, H. J. Phys. Chem. 1978, 82, 371-372. (c) Barata-Vallejo, S.; Flesia, M. M.; Lantaño, B.; Argüello, J. E.; Peñéñory, A. B.; Postigo, A. Eur. J. Org. Chem. 2013, 2013, 998-1008. (d) Franz, J. F.; Kraus, W. B.; Zeitler, K. Chem. Commun. 2015, 51, 8280-8283.

(24) An electronically excited state possesses a much lower ionization potential (i.e., it is a better reductant) than the ground state; see: Turro, N. J.; Ramamurthy, V.; Scaiano, J. C. Energy transfer and electron transfer. Modern Molecular Photochemistry of Organic Molecules; University Science Books: Sausalito, CA, 2010; Chapter 7, p 383. Since an excited state has an inherent propensity to form a supramolecular complex, the generation of an exciplex between the excited enamine $I^{*}$ and bromomalonate $2 \mathrm{c}$ cannot be excluded; however, we could not observe any emission diagnostic of possible excited-state aggregations.

(25) (a) Studer, A.; Curran, D. P. Angew. Chem., Int. Ed. 2016, 55, 58-102. (b) Kärkäs, M. D.; Matsuura, B. S.; Stephenson, C. R. J. Science 2015, 349, 1285-1286.

(26) (a) Ismaili, H.; Pitre, S. P.; Scaiano, J. C. Catal. Sci. Technol. 2013, 3, 935-937. (b) Wayner, D. D. M.; Dannenberg, J. J.; Griller, D. Chem. Phys. Lett. 1986, 131, 189-191.

(27) Julliard, M.; Chanon, M. Chem. Rev. 1983, 83, 425-506.

(28) For a process in which one photon produces only one molecule of the product, the quantum yield can be a maximum of 1 , while for a chain process where one photon forms $n$ molecules of product, the quantum yield is expected to be $>1$. However, a value of $\Phi<1$ does not exclude a possible radical chain mechanism. This is because of potential nonproductive energy-wasting photochemical processes.

(29) Both the quantum yield measurements and the kinetic experiments were performed in acetonitrile to avoid the precipitation of the lutidinium bromide salt generated during the reaction (see ref 12), which is insoluble in MTBE instead. The presence of a precipitate would scatter the irradiating light, thus precluding a reliable measurement.

(30) Yoon recently reported that a related enamine-mediated alkylation of octanal with bromomalonate $2 \mathrm{c}$ using a polypyridylruthenium(II) complex as an external photoredox catalyst possesses a similar quantum yield $(\Phi=18)$, further indicating a radical chain mechanism; see: Cismesia, M. A.; Yoon, T. P. Chem. Sci. 2015, 6, $5426-5434$

(31) Nonproductive processes can reduce the efficiency of photoinitiation $\left(\Phi_{\text {initiation }}\right)$, directly affecting the overall quantum yield $\left(\Phi_{\text {measured }}\right)$. For example, the photoexcited enamine could relax to the ground state via either radiative or vibrational pathways without undergoing electron-transfer processes, while a back electron transfer (BET) from the chiral ion pair III (Figure 3d) would unproductively restore the ground-state enamine-based EDA complex II.

(32) The quantum yield of the initiation step $\left(\Phi_{\text {initiation }}\right)$ was measured by monitoring the decomposition of catalyst $\mathbf{A}$. The analysis is based on the observation (extensively discussed in a later section of the paper) that the enamine I serves as a sacrificial initiator of the chain mechanism: for any photoinduced SET event, a propagating radical, IV, is generated while a molecule of the chiral catalyst $\mathbf{A}$ is destroyed via decomposition of the intermediate $\mathbf{V}$ (Figures $3 \mathrm{~d}$ and 5). If a molecule of the catalyst $\mathbf{A}$ decomposes for any photoinduced initiation, the rate of catalyst degradation correlates with the efficiency of the initiation. This analysis requires that the catalyst $\mathbf{A}$ degradation is triggered by productive photochemical initiation only, and not by 
other secondary reactions, a scenario which is consistent with experimental observations.

(33) The two photochemical initiation manifolds can both be operative in the alkylations of butanal with $\mathbf{2 a}$ and $\mathbf{2} \mathbf{b}$, but only when using a light that can be absorbed by the transiently generated enamine I $(\lambda<415 \mathrm{~nm})$. Both kinetic experiments and quantum yield measurements with $\mathbf{2 a}$ and $\mathbf{2} \mathbf{b}$ have been conducted using higher wavelengths of irradiation, to avoid any possible contribution from the direct photoexcitation of enamines. Under these conditions, only the EDA complex activation is a viable strategy to generate radicals from $\mathbf{2 a}$ and $\mathbf{2 b}$.

(34) Similar atom-transfer mechanisms have been invoked for the alkylation of enol ethers and enamides with electrophilic radicals; see:

(a) Curran, D. P.; Ko, S.-B. Tetrahedron Lett. 1998, 39, 6629-6632.

(b) Friestad, G. K.; Wu, Y. Org. Lett. 2009, 11, 819-822. This pathway was also proposed in ref $25 \mathrm{a}, \mathrm{p} 80$.

(35) (a) Kharasch, M. S.; Jensen, E. V.; Urry, W. H. Science 1945, 102, 128. (b) Pintauer, T.; Matyjaszewski, K. Encyclopedia of Radicals; Wiley: Hoboken, NJ, 2012; Vol. 4, p 1851.

(36) (a) Kornblum, N. Angew. Chem., Int. Ed. Engl. 1975, 14, 734745. (b) Rossi, R. A.; Pierini, A. B.; Peñéñory, A. B. Chem. Rev. 2003, 103, 71-168.

(37) Russell, G. A.; Wang, K. J. Org. Chem. 1991, 56, 3475-3479.

(38) As suggested by a reviewer, deprotonation of an acidic $\alpha$-proton within the $\alpha$-iminyl radical cation $\mathbf{V}$ to form an intracyclic iminium ion is a likely degradation pathway.

(39) Interestingly, an analogous sacrificial role of the enamine was proposed to support the mechanism of the alkylation of aldehydes with bromomalonate $2 \mathrm{c}$, using a dual photoredox-organocatalytic system; see ref 7a.

(40) By using an internal standard and in situ NMR spectroscopic analysis, we confirmed that the overall amount of the catalyst A decreased over time, further supporting the instability of the intermediate $\mathbf{V}$ generated upon photoinduced SET. Studies on the disappearance of the catalyst are reported in Figure 10. The same studies were repeated using gas chromatography (GC-FID); details are reported in section $\mathrm{F}$ in the Supporting Information.

(41) ${ }^{1} \mathrm{H}$ NMR spectroscopic analysis did not allow us to discriminate between the free enamine $I$ and the enamine which engages in EDA complex formation with $\mathbf{2 a}$. The reported I:A ratio refers to the total amount of enamine I present in solution.

(42) For the description and use of a similar illuminating device, based on LED, see: (a) Feldmeier, C.; Bartling, H.; Riedle, E.; Gschwind, R. M. J. Magn. Reson. 2013, 232, 39-44. (b) Feldmeier, C.; Bartling, H.; Magerl, K.; Gschwind, R. M. Angew. Chem., Int. Ed. 2015, 54, 1347-1351.

(43) Initial-rate kinetic studies on the alkylation of 1a with phenacyl bromide $\mathbf{2 b}$ gave a profile very similar to that of the alkylation with $\mathbf{2 a}$ (Figure 9a).This is consistent with the EDA complex activation being the underlying photochemical initiation for both systems. Kinetic studies for the reaction with $\mathbf{2 b}$ are detailed in section $\mathrm{I} 2$ of the Supporting Information.

(44) We could not use the method of reaction progress kinetic analysis to provide a rapid and comprehensive kinetic profile of the reactions because of the significant catalyst degradation pathway. For an overview highlighting the potential of this approach, see: Blackmond, D. G. Angew. Chem., Int. Ed. 2005, 44, 4302-4320.

(45) For a similar treatment of kinetic data for a reaction proceeding through a radical chain mechanism, see: Boisvert, L.; Denney, M. C.; Hanson, S. K.; Goldberg, K. I. J. Am. Chem. Soc. 2009, 131, 1580215814 .

(46) When monitoring the progress of the alkylation with $2 \mathrm{c}$ using in situ NMR methodology, we did observe catalyst degradation. This is presumably because of decomposition pathways triggered by the presence of oxygen in the system, as in situ monitoring did not allow for complete deoxygenation of the reaction medium. 